\title{
Microwave mediated syntheses of $\beta$-enamino thioic acid derivatives
}

\author{
Alan R. Katritzky,* Hui Tao, and Kostyantyn Kirichenko \\ Center for Heterocyclic Compounds, Department of Chemistry, University of Florida, \\ Gainesville, FL 32611-7200 \\ E-mail: katritzky@chem.ufl.edu
}

\section{Submitted to honor the $65^{\text {th }}$ Anniversary of Professor Alain Krief}

\begin{abstract}
Reaction of di(benzotriazole-1-yl)methanethione $\mathbf{1}$ with imines $\mathbf{2 a - f}$ gave air and moisture stable benzotriazolyl $\beta$-enaminothiones 3a-f. The thioacylbenzotriazoles $3 \mathbf{a}-\mathbf{f}$ enable simple and efficient preparation of $\beta$-enamino thioic acid derivatives (thioamides, thioesters and dithioesters) in 74-99\% yields via microwave mediated nucleophilic substitution of the benzotriazolyl moiety. C-Thioacylation with 1-thioacyl-6-nitrobenzotriazoles $\mathbf{7 a - c}$ is also discussed.
\end{abstract}

Keywords: Microwave, benzotriazole, $\beta$-enaminothiones, 1-thioacyl-6-nitrobenzotriazoles, C-thioacylation

\section{Introduction}

Organosulfur compounds possess a rich and varied chemistry, and diverse biological properties. ${ }^{1,2,3}$ Among them, $\beta$-enaminothioic acids are important building blocks for heterocycle construction e.g., pyrazoles, ${ }^{4} 4$-aminoquinolines, ${ }^{5}$ dihydrothiopyrans, ${ }^{6,7}$ thiazoline, ${ }^{8}$ thiazolin-4one, ${ }^{8} 1,3$-thiazolin-4-one, ${ }^{8} 6 H$-1,3-thiazines, ${ }^{9}$ as well as useful precursors for liquid crystals ${ }^{10}$ and $\beta$-keto thioic acid derivatives. ${ }^{11} \beta$-Enaminothioic acids are reported as good 1-thia-1,3dienes ${ }^{\text {Error! Bookmark not defined., } 12,13,14}$ and Michael acceptors. ${ }^{15}$

Despite the importance of $\beta$-enaminothioic acids, existing methods for their preparation are limited to: i) the reaction of $\beta$-enaminones with phosphorus pentasulfide or other $\mathrm{O} / \mathrm{S}$ exchange reagents, such as Lawesson's reagents; ${ }^{16}$ ii) the cycloaddition of unactivated 2-aza-1,3-dienes to isothiocyanates to give 1,2-dihydropyrimidin-4(3H)-thiones, followed by reduction with $\mathrm{LiAlH}_{4}$ to provide $\beta$-enamino thioamides; ${ }^{11}$ iii) the reaction of $\beta$-enaminones with aryl isothiocyanates at $90{ }^{\circ} \mathrm{C} ;{ }^{17,18}$ and iv) the reaction of cyclopentanones and 2 -substituted cyclopentanones with 
carbon disulphide and ammonia at $0{ }^{\circ} \mathrm{C} .{ }^{19}$ The first method involves foul smelling starting materials and/or intermediates, while the other methods are limited to specific substrates.

We now disclose a novel and efficient synthetic protocol for benzotriazolyl enaminothiones 3a-f from dibenzotriazolylmethanethione $\mathbf{1}$ and the application of these products to the preparation of $\beta$-enamino thioamides $4 \mathbf{a}-\mathbf{c}$, thioesters $\mathbf{5 a}-\mathbf{c}$, and dithioesters $\mathbf{6 a}-\mathbf{c}$.

\section{Results and Discussion}

The reaction of thiophosgene with four equivalents of benzotriazole in methylene chloride at $0{ }^{\circ} \mathrm{C}$ gave dibenzotriazolylmethanethione 1 in $87 \%$ yield (Scheme 1$){ }^{20}$

The use of twofold excess of benzotriazole advantageously avoids the precursory generation of either 1-trimethylsilyl benzotriazole ${ }^{21}$ or the sodium salt of benzotriazole, ${ }^{20}$ required in the previously reported protocols. Excess of benzotriazole and low reaction temperature appear to be essential for successful preparation of $\mathbf{1} .^{20}$

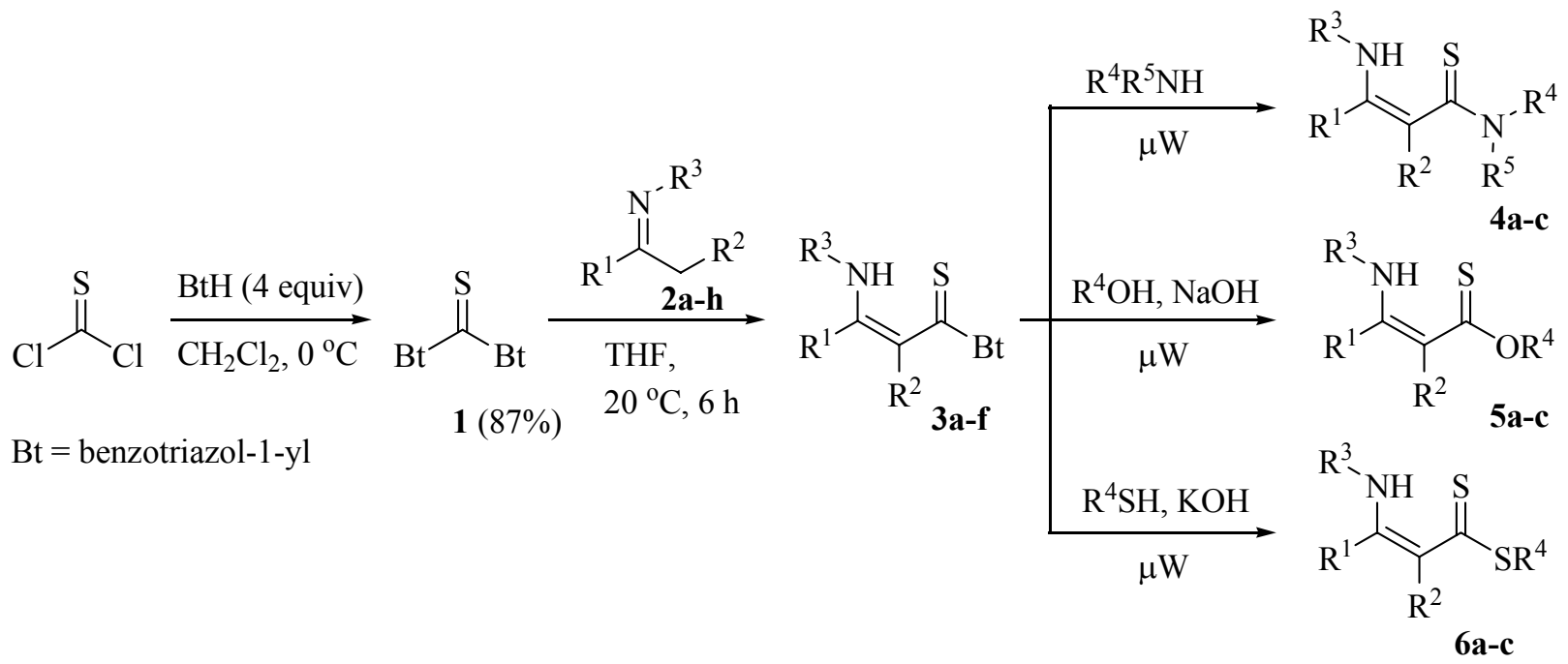

\section{Scheme 1}

Dibenzotriazolylmethanethione $\mathbf{1}$ reacted with equimolar ketimines $\mathbf{2 a}-\mathbf{f}$ in $\mathrm{THF}$ at $20{ }^{\circ} \mathrm{C}$ to give benzotriazolyl enaminothiones 3a-f (78-97\%) (Table 1). The treatment of 1 with a twofold excess of ketimine $\mathbf{2 a}-\mathbf{f}$ resulted in exclusive formation of $\mathbf{3 a}-\mathbf{f}$. Attempted reactions of $\mathbf{1}$ with aldimines $\mathbf{2 g}, \mathbf{h}$ and 1-cyclohexenylpyrrolidine (not shown) failed.

Structures 3a-f were supported by their ${ }^{1} \mathrm{H}$ and ${ }^{13} \mathrm{C}$ NMR spectra, and elemental analyses (see Experimental Section). In the ${ }^{1} \mathrm{H}$ NMR spectra of benzotriazolyl enaminothiones 3a-f, the broad singlet signals in the range 13.21-14.95 ppm and the singlet signals at 7.16-7.21 ppm (for compounds 3a,b,e) corresponding to $\mathrm{NH}$ and $\mathrm{CH}$ of enamine fragment, respectively, confirmed exclusive existence in the Z-enamine form, resulting from $\mathrm{N} \cdots \mathrm{H} \cdots \mathrm{S}$ chelation. 
Treatment of compounds $\mathbf{3 a}, \mathbf{b}$ under microwave irradiation at $80{ }^{\circ} \mathrm{C}$ with secondary amines gave $\beta$-enamino thioamides 4a-c (92-95\%) (Scheme 1, Table 1). Similar treatment of 3a,b with alcohols or thiols in the presence of sodium or potassium hydroxide afforded thioesters 5a-c (74-99\%) and dithioesters 6a-c (85-92\%), respectively.

Table 1. Microwave-mediated synthesis of $\beta$-enamino thioic acid derivatives 3-6

\begin{tabular}{|c|c|c|c|c|c|c|c|}
\hline Entry & Product & $\mathrm{R}^{1}$ & $\mathrm{R}^{2}$ & $\mathrm{R}^{3}$ & $\mathrm{R}^{4}$ & $\mathrm{R}^{5}$ & Yield, $\%$ \\
\hline 1 & $3 \mathbf{a}$ & $\mathrm{Ph}$ & $\mathrm{H}$ & $\mathrm{Bu}$ & - & - & 95 \\
\hline 2 & 3b & 4-Py & $\mathrm{H}$ & $\mathrm{Bu}$ & - & - & 94 \\
\hline 3 & $3 c$ & $\mathrm{Ph}$ & $\mathrm{Me}$ & $\mathrm{Bu}$ & - & - & 83 \\
\hline 4 & 3d & \multicolumn{2}{|c|}{$-\left(\mathrm{CH}_{2}\right)_{4-}$} & $\mathrm{Bu}$ & - & - & 78 \\
\hline 5 & $3 e$ & \multicolumn{2}{|c|}{$-\left(\mathrm{CH}_{2}\right)_{4}-$} & $\mathrm{Bn}$ & - & - & 95 \\
\hline 6 & $3 f$ & Et & $\mathrm{H}$ & $\mathrm{Bu}$ & - & - & 78 \\
\hline 7 & $3 g$ & $\mathrm{H}$ & vinyl & $\mathrm{Ph}$ & - & - & 0 \\
\hline 8 & $3 \mathbf{h}$ & $\mathrm{H}$ & Et & $\mathrm{Bn}$ & - & - & 0 \\
\hline 9 & $4 a$ & $\mathrm{Ph}$ & $\mathrm{H}$ & $\mathrm{Bu}$ & \multicolumn{2}{|c|}{$-\mathrm{C}_{2} \mathrm{H}_{4} \mathrm{OC}_{2} \mathrm{H}_{4}-$} & 91 \\
\hline 10 & $4 b$ & $\mathrm{Ph}$ & $\mathrm{H}$ & $\mathrm{Bu}$ & \multicolumn{2}{|c|}{$-\left(\mathrm{CH}_{2}\right)_{5}-$} & 95 \\
\hline 11 & $4 c$ & 4-Py & $\mathrm{H}$ & $\mathrm{Bu}$ & \multicolumn{2}{|c|}{$-\mathrm{C}_{2} \mathrm{H}_{4} \mathrm{OC}_{2} \mathrm{H}_{4}-$} & 92 \\
\hline 12 & $5 \mathbf{a}$ & $\mathrm{Ph}$ & $\mathrm{H}$ & $\mathrm{Bu}$ & $\mathrm{Me}$ & - & 95 \\
\hline 13 & $5 b$ & $\mathrm{Ph}$ & $\mathrm{H}$ & $\mathrm{Bu}$ & $\operatorname{Pr}$ & - & 94 \\
\hline 14 & $5 c$ & 4-Py & $\mathrm{H}$ & $\mathrm{Bu}$ & $\mathrm{Me}$ & - & 74 \\
\hline 15 & $6 a$ & $\mathrm{Ph}$ & $\mathrm{H}$ & $\mathrm{Bu}$ & $n-\mathrm{C}_{6} \mathrm{H}_{13}$ & - & 85 \\
\hline 16 & $6 b$ & $\mathrm{Ph}$ & $\mathrm{H}$ & $\mathrm{Bu}$ & $\mathrm{Ph}$ & - & 87 \\
\hline 17 & $6 c$ & 4-Py & $\mathrm{H}$ & $\mathrm{Bu}$ & $\mathrm{Ph}$ & - & 92 \\
\hline
\end{tabular}

As with compounds 3a,b, the ${ }^{1} \mathrm{H}$ NMR spectra of products 4-6 showed the presence of a broad singlet in the range 11.44-13.16 ppm corresponding to $\mathrm{NH}$ proton suggesting exclusive Zenaminothione configuration (Scheme 1).

However, under the same conditions, treatment of benzotriazolyl enaminothiones $\mathbf{3 c}-\mathbf{f}$ with alcohols, thiols or amines resulted in the recovery of $\mathbf{3 c}-\mathbf{f}$. Attempted treatments of $\mathbf{3 c}-\mathbf{f}$ with sodium methoxide in methanol under microwave irradiation caused decomposition of the starting materials, while no reaction occurred upon stirring at room temperature for $48 \mathrm{~h}$.

When group $\mathrm{R}^{1}$ is aryl or heteroaryl group (compounds $\mathbf{3 a}, \mathbf{b}$ ), which behave as electron withdrawing groups, the electrophilicity of thioacyl group increases, making the benzotriazolyl $\beta$-enaminothione reactive toward secondary amines, alcohols and thiols. However, when $\mathrm{R}^{1}$ is an alkyl group (compounds 3d-f), which behave as electron donating groups, the electrophilicity of the thioacyl group decreases, resulting in no reaction under same reaction conditions. Compound 3c was also unreactive toward secondary amines, alcohols and thiols.

The attempted reaction of benzotriazolyl enaminothione 3a with hydrazine under microwave irradiation at $80^{\circ} \mathrm{C}$ failed and resulted in a complex set of polar products. Treatment of $\mathbf{3 a}$ with 
nitromethane or acetonitrile at $20^{\circ} \mathrm{C}$ or heating up to $80{ }^{\circ} \mathrm{C}$ under microwave irradiation in the presence of sodium hydroxide gave no reaction.

\section{Synthesis of $\beta$-enaminothiones}

Thioacyl-6-nitrobenzotriazoles 7a-c reacted with ketimine $\mathbf{2 a}$ in THF in the presence of $\mathrm{ZnBr}_{2}$ at $20{ }^{\circ} \mathrm{C}$ for $\mathbf{3} \mathrm{d}$ to give $\beta$-enamino thiones 8a-c in moderate to good yield (Scheme 2). Unfortunately, similar reactions of thioacylbenzotriazoles $7 \mathbf{a}$ with ketimine $\mathbf{2 i}$ produced under the same reaction conditions thioamide 9 in low 35\% yield instead of the expected $\beta$-enamino thione $\mathbf{8}$ (Scheme 2), while the reactions with imines $\mathbf{2 e}$ and $\mathbf{2 h}$ resulted in complex mixtures of products. It is possible that the formation of complex mixtures is due to the concurrent addition of ionized thioacylbenzotriazole 7 to the imine bond, followed by hydrolysis to thioamides, such as 9.

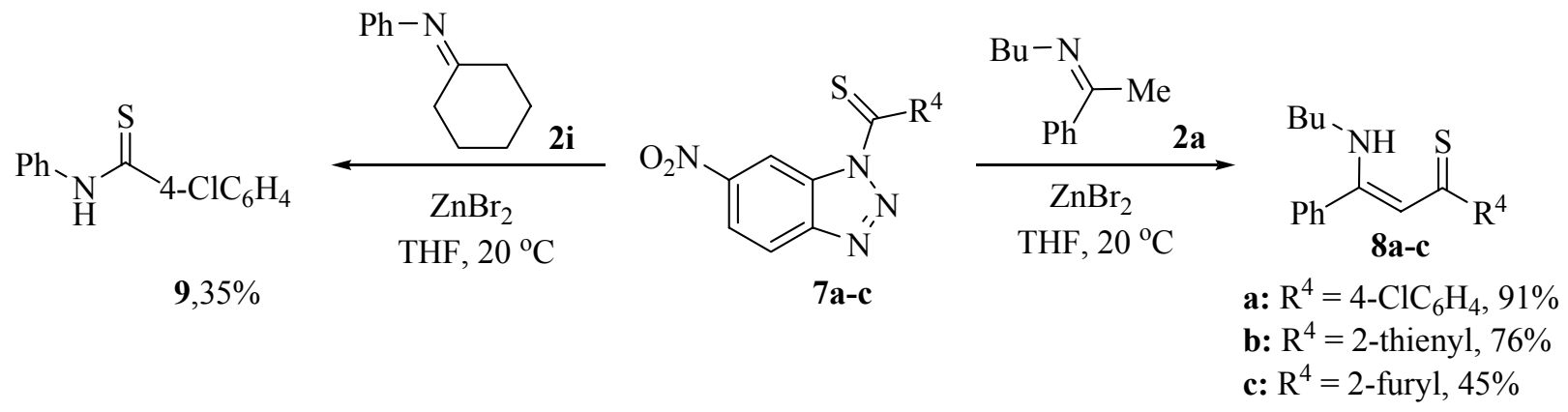

\section{Scheme 2}

Structures 8a-c were supported by their ${ }^{1} \mathrm{H}$ and ${ }^{13} \mathrm{C}$ NMR spectra, and by elemental analyses (see Experimental Section).

The order of addition significantly influences the chemical yield. Thus, the best results were obtained when the appropriate thioacylbenzotriazole 7 in THF was first treated with $\mathrm{ZnBr}_{2}$, followed by slow addition of the corresponding imine $\mathbf{2}$, in contrast to low yields upon the initial addition of the Lewis acid to a solution of ketimine $\mathbf{2} \mathbf{a}$ in THF.

After screening a series of nucleophiles, including Grignard, organozinc, organolithium reagents, enolates, silyl enol ethers, allyl trimethyl silane, and active methylenes, enamines and aldimines, ketimines were the only nucleophiles found to be effectively thioacylated by 1 thioacylbenzotriazoles 7 .

\section{Conclusions}

In conclusion, a novel and general approach to $\beta$-enamino thioic acid derivatives has been developed. The procedure described appears to be general and represents an efficient, simple and alternative route to $\beta$-enamino thioic acid derivatives 4-6. 


\section{Experimental Section}

General Procedures. Melting points were determined on a hot-stage apparatus equipped with a digital thermometer and are uncorrected. NMR spectra were recorded on a Varian Gemini 300 spectrometer in $\mathrm{CDCl}_{3}$ with tetramethylsilane as the internal standard for ${ }^{1} \mathrm{H}(300 \mathrm{MHz})$ or solvent as the internal standard for ${ }^{13} \mathrm{C}(75 \mathrm{MHz})$ unless otherwise stated. The elemental analyses were performed on a Carlo Erba EA-1108 instrument. Anhydrous THF was used freshly distilled from sodium/benzophenone. Column chromatography was conducted on silica gel 200-245 meshes.

Imines 2a-i were prepared according to the published procedures: butyl(1phenylethylidene)amine (2a), colorless oil (91\%); ${ }^{22}$ butyl[1-(pyridin-4-yl)ethylidene]amine (2b), colorless oil $\quad(78 \%) ;{ }^{23}$ butyl(1-phenylpropylidene)amine $\quad(\mathbf{2 c})$, colorless oil $(88 \%) ;{ }^{24}$ butyl(cyclohexylidene)amine (2d), colorless oil $(83 \%) ;{ }^{25}$ benzyl(cyclohexylidene)amine (2e), colorless oil $(85 \%) ;{ }^{26}$ butyl(butan-2-ylidene)amine (2f), colorless oil $(88 \%){ }^{22} \mathrm{~N}-(3-$ butenylidene)aniline (2g), colorless oil (73\%); benzyl(butylidene)amine (2h), colorless oil $(78 \%),{ }^{27} \mathrm{~N}$-(cyclohexylidene)aniline (2i), colorless oil $(61 \%){ }^{28}$

1-Thioacyl-6-nitrobenzotriazoles 7a-c were prepared according to published procedures: ${ }^{29}$ (6-nitrobenzotriazol-1-yl)(2-thienyl)methanethione (7b), gray microcrystals (40\%), mp 132-134 ${ }^{\circ} \mathrm{C}$ (lit. ${ }^{29} \mathrm{mp} 133-134 \quad{ }^{\circ} \mathrm{C}$ ); (6-nitrobenzotriazol-1-yl)(2-furyl)methanethione (7c), orange microcrystals $(80 \%)$, $\mathrm{mp} 162-163{ }^{\circ} \mathrm{C}\left(\right.$ lit. $\left.^{29} \mathrm{mp} 161-162{ }^{\circ} \mathrm{C}\right)$.

(6-Nitrobenzotriazol-1-yl)-4-chlorophenylmethanethione (7a). Recrystallized from hexanes to give pink microcrystals $(93 \%), \mathrm{mp} 161-163{ }^{\circ} \mathrm{C} ;{ }^{1} \mathrm{H}$ NMR $\delta 9.49(\mathrm{~d}, J=1.9 \mathrm{~Hz}, 1 \mathrm{H}), 8.48(\mathrm{dd}, J$ $=8.9,2.1 \mathrm{~Hz}, 1 \mathrm{H}), 8.34(\mathrm{~d}, J=8.9 \mathrm{~Hz}, 1 \mathrm{H}), 7.76(\mathrm{~d}, J=8.7 \mathrm{~Hz}, 2 \mathrm{H}), 7.48(\mathrm{~d}, J=8.7 \mathrm{~Hz}, 2 \mathrm{H})$; ${ }^{13} \mathrm{C}$ NMR $\delta 199.4,149.1,140.2,132.9,132.1,128.7,121.9,121.4,112.2$. Anal. Calcd for $\mathrm{C}_{13} \mathrm{H}_{7} \mathrm{ClN}_{4} \mathrm{O}_{2} \mathrm{~S}: \mathrm{C}, 48.99 ; \mathrm{H}, 2.21 ; \mathrm{N}, 17.58$. Found: $\mathrm{C}, 49.11 ; \mathrm{H}, 2.19 ; \mathrm{N}, 16.22$.

Procedure for the preparation of dibenzotriazolylmethanethione (1). Thiophosgene (11.5 g, $10 \mathrm{mmol}$ ) was added dropwise to a solution of benzotriazole $(4.77 \mathrm{~g}, 40 \mathrm{mmol})$ in dichloromethane $(50 \mathrm{~mL})$ at $0{ }^{\circ} \mathrm{C}$. The reaction mixture was stirred at the same temperature for $3 \mathrm{~h}$. The mixture was filtered, and the solid residue was washed with dichloromethane $(3 \times 30$ $\mathrm{mL})$. The filtrate was washed with $5 \%$ aqueous $\mathrm{Na}_{2} \mathrm{CO}_{3}(3 \times 50 \mathrm{~mL})$. The solvent was removed under vacuum to dryness, and the residue was recrystallized from dichloromethane to give di(1H-benzotriazol-1-yl)methanethione in $87 \%$ yield as yellow microcrystals, $\mathrm{mp} 171{ }^{\circ} \mathrm{C}$ (lit. ${ }^{21} \mathrm{mp}$ $\left.170-172{ }^{\circ} \mathrm{C}\right)$.

\section{General procedure for the preparation of benzotriazolyl $\beta$-enaminothiones 3a-h}

To a solution of dibenzotriazolylmethanethione 1 (280 mg, $1 \mathrm{mmol})$ in THF $(50 \mathrm{~mL})$, appropriate imine $2(1 \mathrm{mmol})$ was added at room temperature. The reaction mixture was stirred at the same temperature for $6 \mathrm{~h}$, and then concentrated under vacuum. The residue was dissolved in ethyl acetate $(50 \mathrm{~mL})$, and was washed with $5 \%$ aqueous $\mathrm{Na}_{2} \mathrm{CO}_{3}(3 \times 30 \mathrm{~mL})$, followed by 
brine $(30 \mathrm{~mL})$. The organic layer was dried over anhydrous $\mathrm{Na}_{2} \mathrm{SO}_{4}$, and the solvent was removed under vacuum. The residue was either recrystallized from dichloromethane/hexanes or purified by flash chromatography (hexanes/ethyl acetate $5: 1$ ) on silica gel to give $\mathbf{3 a}-\mathbf{h}$.

(Z)-1-(1H-Benzotriazol-1-yl)-3-(butylamino)-3-phenyl-2-propene-1-thione (3a). Recrystallized from dichloromethane to give yellow microcrystals (95\%), mp 94-96 ${ }^{\circ} \mathrm{C} ;{ }^{1} \mathrm{H}$ NMR $\delta 13.36$ (br s, $1 \mathrm{H}), 8.82(\mathrm{~d}, J=8.5 \mathrm{~Hz}, 1 \mathrm{H}), 8.06(\mathrm{~d}, J=8.2 \mathrm{~Hz}, 1 \mathrm{H}), 7.58-7.38(\mathrm{~m}, 7 \mathrm{H}), 7.21(\mathrm{~s}, 1 \mathrm{H})$, 3.47-3.40 (m, 2H), 1.73-1.64 (m, 2H), 1.53-1.41 (m, 2H), $0.93(\mathrm{t}, J=7.3 \mathrm{~Hz}, 3 \mathrm{H}) ;{ }^{13} \mathrm{C}$ NMR $\delta$ 179.3, 169.4, 147.0, 135.1, 132.5, 130.3, 128.8, 128.5, 127.3, 124.7, 119.8, 115.7, 106.5, 45.7, 32.1, 19.9, 13.5. Anal. Calcd for $\mathrm{C}_{19} \mathrm{H}_{20} \mathrm{~N}_{4} \mathrm{~S}$ : C, 67.83; H, 5.99; N, 16.65. Found: C, 67.80; H, 6.04; N, 16.74.

(Z)-1-(1H-Benzotriazol-1-yl)-3-(butylamino)-3-(4-pyridinyl)-2-propene-1-thione (3b). Recrystallized from hexanes to give yellow microcrystals (94\%), mp 100-102 ${ }^{\circ} \mathrm{C} ;{ }^{1} \mathrm{H}$ NMR $\delta 13.21$ (br s, $1 \mathrm{H}$ ), 8.85-8.80 (m, 3H), 8.07 (d, $J=8.2 \mathrm{~Hz}, 1 \mathrm{H}), 7.61-7.56(\mathrm{~m}, 1 \mathrm{H}), 7.46-7.38(\mathrm{~m}, 3 \mathrm{H}), 7.19(\mathrm{~s}, 1 \mathrm{H})$, $3.40-3.34(\mathrm{~m}, 2 \mathrm{H}), 1.73-1.64(\mathrm{~m}, 2 \mathrm{H}), 1.54-1.42(\mathrm{~m}, 2 \mathrm{H}), 0.95(\mathrm{t}, J=7.3 \mathrm{~Hz}, 3 \mathrm{H}) ;{ }^{13} \mathrm{C}$ NMR $\delta$ $181.5,165.8,150.6,147.0,142.8,132.5,129.0,125.0,121.7,120.0,115.7,104.8,45.6,32.1$, 19.9, 13.5. Anal. Calcd for $\mathrm{C}_{18} \mathrm{H}_{19} \mathrm{~N}_{5} \mathrm{~S}$ : C, 64.07; H, 5.68; N, 20.75. Found: C, 64.33; H, 5.69; N, 20.72 .

(Z)-1-(1H-Benzotriazol-1-yl)-3-(butylamino)-2-methyl-3-phenyl-2-propene-1-thione (3c). Orange oil (83\%); ${ }^{1} \mathrm{H}$ NMR $\delta 15.22$ (br s, $\left.1 \mathrm{H}\right), 8.04(\mathrm{~d}, J=8.5 \mathrm{~Hz}, 1 \mathrm{H}), 7.87(\mathrm{~d}, J=8.5 \mathrm{~Hz}, 1 \mathrm{H})$, 7.56-7.46 (m, 4H), 7.39-7.27 (m, 3H), 3.28-3.22 (m, 2H), 1.71-1.60 (m, 2H), 1.51-1.35 (m, $5 \mathrm{H}), 0.90(\mathrm{t}, J=7.0 \mathrm{~Hz}, 3 \mathrm{H}) ;{ }^{13} \mathrm{C}$ NMR $\delta 175.0,172.6,145.3,133.2,132.0,129.5,129.0,127.4$, $125.9,123.7,119.1,115.5,112.1,46.2,31.4,19.7,19.2$, 13.2. Anal. Calcd for $\mathrm{C}_{20} \mathrm{H}_{22} \mathrm{~N}_{4} \mathrm{~S}: \mathrm{C}$, 68.54; H, 6.33; N, 15.99. Found: C, 68.68; H, 6.40; N, 15.62.

1H-Benzotriazol-1-yl[2-(butylamino)-1-cylohexen-1-yl]methanethione (3d). Viscous red oil (78\%); ${ }^{1} \mathrm{H}$ NMR $\delta 14.94$ (br s, $\left.1 \mathrm{H}\right), 8.05$ (d, $\left.J=8.5 \mathrm{~Hz}, 1 \mathrm{H}\right), 7.77$ (d, $\left.J=8.2 \mathrm{~Hz}, 1 \mathrm{H}\right), 7.49$ (t, $J=$ $7.6 \mathrm{~Hz}, 1 \mathrm{H}), 7.39-7.34(\mathrm{~m}, 1 \mathrm{H}), 3.57-3.50(\mathrm{~m}, 2 \mathrm{H}), 2.67(\mathrm{t}, J=6.7 \mathrm{~Hz}, 2 \mathrm{H}), 2.20(\mathrm{t}, J=6.4 \mathrm{~Hz}$, 2H), $1.89-1.74(\mathrm{~m}, 4 \mathrm{H}), 1.66-1.56(\mathrm{~m}, 2 \mathrm{H}), 1.52-1.44(\mathrm{~m}, 2 \mathrm{H}), 1.02(\mathrm{t}, J=7.3 \mathrm{~Hz}, 3 \mathrm{H}) ;{ }^{13} \mathrm{C}$ NMR $\delta 173.0,171.5,145.2,131.8,127.3,123.7,119.1,118.5,111.8,43.8,30.3,27.8,27.5,21.8$, 20.7, 20.0, 13.4. Anal. Calcd for $\mathrm{C}_{17} \mathrm{H}_{22} \mathrm{~N}_{4} \mathrm{~S}$ : C, 64.93; H, 7.05; N, 17.82. Found: C, 65.14; H, $7.29 ; \mathrm{N}, 18.17$.

1H-Benzotriazol-1-yl[2-(benzylamino)-1-cyclohexen-1-yl]methanethione(3e). Recrystallized from dichloromethane//hexanes to give orange microcrystals $(95 \%), \mathrm{mp} 89-91{ }^{\circ} \mathrm{C}$; ${ }^{1} \mathrm{H}$ NMR $\delta$ 15.24 (br s, 1H), 8.07 (d, $J=8.2 \mathrm{~Hz}, 1 \mathrm{H}), 7.78(\mathrm{~d}, J=8.6 \mathrm{~Hz}, 1 \mathrm{H}), 7.52-7.36(\mathrm{~m}, 7 \mathrm{H}), 4.73(\mathrm{~d}, J$ $=5.8 \mathrm{~Hz}, 2 \mathrm{H}), 2.67(\mathrm{t}, J=6.7 \mathrm{~Hz}, 2 \mathrm{H}), 2.21(\mathrm{t}, J=6.4 \mathrm{~Hz}, 2 \mathrm{H}), 1.76-1.68(\mathrm{~m}, 2 \mathrm{H}), 1.50-1.42$ $(\mathrm{m}, 2 \mathrm{H}) ;{ }^{13} \mathrm{C}$ NMR $\delta 175.2,171.9,145.4,134.7,132.2,129.2,128.3,127.8,127.5,124.2,119.4$, 118.9, 112.2, 47.8, 28.2, 27.9, 22.0, 20.9. Anal. Calcd for $\mathrm{C}_{20} \mathrm{H}_{20} \mathrm{~N}_{4} \mathrm{~S}: \mathrm{C}, 68.94 ; \mathrm{H}, 5.78 ; \mathrm{N}$, 16.08. Found: C, 68.71; H, 5.90; N, 16.35 .

(Z)-1-(1H-Benzotriazol-1-yl)-3-(butylamino)-2-pentene-1-thione (3f). Recrystallized from hexanes to give light yellow microcrystals (78\%), mp 58-60 ${ }^{\circ} \mathrm{C} ;{ }^{1} \mathrm{H}$ NMR $\delta 13.30$ (br s, $1 \mathrm{H}$ ), $8.77(\mathrm{~d}, J=8.2 \mathrm{~Hz}, 1 \mathrm{H}), 8.06$ (d, $J=8.2 \mathrm{~Hz}, 1 \mathrm{H}), 7.56-7.51(\mathrm{~m}, 1 \mathrm{H}), 7.43-7.37$ (m, 1H), 7.16 
(s, 1H), 3.57-3.51 (m, 2H), 2.55 (q, $J=7.6 \mathrm{~Hz}, 2 \mathrm{H}), 1.82-1.75(\mathrm{~m}, 2 \mathrm{H}), 1.65-1.54(\mathrm{~m}, 2 \mathrm{H}), 1.31$ $(\mathrm{t}, J=7.6 \mathrm{~Hz}, 3 \mathrm{H}), 1.03(\mathrm{t}, J=7.3 \mathrm{~Hz}, 3 \mathrm{H}) ;{ }^{13} \mathrm{C}$ NMR $\delta 178.4,173.4,147.0,132.6,128.3,124.6$, 119.7, 115.6, 104.8, 43.6, 31.4, 27.3, 20.2, 13.6, 12.1 .

General procedure for the preparation of $\beta$-enamino thioamides $4 a-c$ from benzotriazolyl 及-enaminothiones 3

Benzotriazolyl $\beta$-enaminothione $3(0.3 \mathrm{mmol})$ was dissolved in secondary amine $(2 \mathrm{~mL})$. The mixture was exposed to microwave irradiation $\left(80\right.$ Watts, $80^{\circ} \mathrm{C}$ ) for $0.5 \mathrm{~h}$. The solvent was removed under vaccuum. The residue was dissolved in dichloromethane $(10 \mathrm{~mL})$ and washed with $5 \%$ aqueous $\mathrm{Na}_{2} \mathrm{CO}_{3}(3 \times 10 \mathrm{~mL})$, followed by brine $(10 \mathrm{~mL})$. The organic layer was dried over anhydrous $\mathrm{Na}_{2} \mathrm{SO}_{4}$. The solvent was removed under vacuum, and the residue was purified by flash chromatography (hexanes/ethyl acetate $5: 1$ ) on silica gel to give $4 a-c$.

(Z)-3-(Butylamino)-1-morpholino-3-phenyl-2-propene-1-thione (4a). Light yellow oil (91\%); ${ }^{1} \mathrm{H}$ NMR $\delta 11.97$ (br s, 1H), 7.43-7.41 (m, 3H), 7.34-7.33 (m, 2H), 5.14 (s, 1H), 3.99-3.91 (m, $4 \mathrm{H}), 3.71(\mathrm{t}, J=5.1 \mathrm{~Hz}, 4 \mathrm{H}), 3.15-3.08(\mathrm{~m}, 2 \mathrm{H}), 1.55-1.48(\mathrm{~m}, 2 \mathrm{H}), 1.41-1.34(\mathrm{~m}, 2 \mathrm{H}), 0.86(\mathrm{t}$, $J=7.4 \mathrm{~Hz}, 3 \mathrm{H}) ;{ }^{13} \mathrm{C}$ NMR $\delta$ 187.0, 164.1, 137.9, 128.9, 128.3, 127.4, 93.3, 66.5, 47.7, 44.6, 32.5, 20.0, 13.6. Anal. Calcd for $\mathrm{C}_{17} \mathrm{H}_{24} \mathrm{~N}_{2} \mathrm{OS}$ : C, 67.07; H, 7.95; N, 9.20. Found: C, 66.80; H, $7.97 ;$ N, 9.08 .

(Z)-3-(Butylamino)-3-phenyl-1-piperidino-2-propene-1-thione (4b). Yellow oil (95\%); ${ }^{1} \mathrm{H}$ NMR $\delta 11.79$ (br s, 1H), 7.42-7.33 (m, 5H), 5.15 (s, 1H), 3.92 (br s, 4H), 3.11-3.05 (m, 2H), 1.69-1.47 (m, 8H), 1.40-1.33 (m, 2H), $0.85(\mathrm{t}, J=7.3 \mathrm{~Hz}, 3 \mathrm{H}) ;{ }^{13} \mathrm{C}$ NMR $\delta 185,7,163.2,138.3$, 128.6, 128.2, 127.6, 93.4, 49.1, 44.5, 32.6, 25.8, 24.6, 20.0, 13.6. Anal. Calcd for $\mathrm{C}_{18} \mathrm{H}_{26} \mathrm{~N}_{2} \mathrm{~S}$ : C, 71.47; H, 8.66; N, 9.26. Found: C, 71.54; H, 8.87; N, 9.33.

(Z)-3-(Butylamino)-1-morpholino-3-(4-pyridinyl)-2-propene-1-thione (4c). Light yellow oil (92\%); ${ }^{1} \mathrm{H}$ NMR $\delta 11.85$ (br s, $\left.1 \mathrm{H}\right), 8.69(\mathrm{~d}, J=6.1 \mathrm{~Hz}, 2 \mathrm{H}), 7.26$ (d, $\left.J=6.4 \mathrm{~Hz}, 2 \mathrm{H}\right), 5.07$ (s, $1 \mathrm{H}), 3.96$ (br s, 4H), 3.74-3.71 (m, 4H), 3.10-3.04 (m, 2H), 1.58-1.48 (m, 2H), 1.43-1.31 (m, 2H), $0.88(\mathrm{t}, J=7.3 \mathrm{~Hz}, 3 \mathrm{H}) ;{ }^{13} \mathrm{C} \mathrm{NMR} \delta 187.7,160.8,150.2,145.6,122.3,93.0,66.5,47.9$, 44.8, 32.6, 20.0, 13.6. Anal. Calcd for $\mathrm{C}_{16} \mathrm{H}_{23} \mathrm{~N}_{3} \mathrm{OS}$ : C, 62.92; H, 7.59; N, 13.76. Found: C, $63.17 ; \mathrm{H}, 7.88 ; \mathrm{N}, 13.38$.

\section{General procedure for the preparation of $\boldsymbol{\beta}$-enamino thioesters $5 \mathrm{a}-\mathrm{c}$}

Benzotriazolyl $\beta$-enaminothione $3(0.3 \mathrm{mmol})$ was dissolved in $2 \mathrm{~N}$ alcoholic sodium hydroxide $(2 \mathrm{~mL})$. The reaction mixture was exposed to microwave irradiation $\left(80 \mathrm{Watts}, 80^{\circ} \mathrm{C}\right)$ for $0.5 \mathrm{~h}$. The solvent was removed under vacuum to dryness. The residue was dissolved in dichloromethane $(10 \mathrm{~mL})$ and the solution was washed with $5 \%$ aqueous $\mathrm{Na}_{2} \mathrm{CO}_{3}(3 \times 10 \mathrm{~mL})$, followed by brine $(10 \mathrm{~mL})$. The organic layer was dried over anhydrous $\mathrm{Na}_{2} \mathrm{SO}_{4}$. the solvent was removed under vacuum, and the residue was purified by flash chromatography (hexanes/ethyl acetate $5: 1$ ) on silica gel to give $\mathbf{5 a}-\mathbf{c}$.

O-Methyl (Z)-3-(butylamino)-3-phenyl-2-propenethioate (5a). Light yellow oil (95\%); ${ }^{1} \mathrm{H}$ NMR $\delta 11.44$ (br s, 1H), 7.43-7.41 (m, 3H), 7.36-7.31 (m, 2H), 5.49 (s, 1H), 3.97 (s, 3H), 3.21 
$(\mathrm{q}, J=6.2 \mathrm{~Hz}, 2 \mathrm{H}), 1.60-1.50(\mathrm{~m}, 2 \mathrm{H}), 1.44-1.34(\mathrm{~m}, 2 \mathrm{H}), 0.87(\mathrm{t}, J=7.3 \mathrm{~Hz}, 3 \mathrm{H}) ;{ }^{13} \mathrm{C} \mathrm{NMR} \delta$ 202.2, 166.0, 136.4, 129.4, 128.4, 127.4, 100.0, 55.1, 44.8, 32.5, 19.9, 13.6. Anal. Calcd for $\mathrm{C}_{14} \mathrm{H}_{19} \mathrm{NOS}$ : C, 67.43; H, 7.68; N, 5.62. Found: C, 67.41; H, 7.59; N, 5.93.

O-Propyl (Z)-3-(butylamino)-3-phenyl-2-propenethioate (5b). Light yellow oil (94\%); ${ }^{1} \mathrm{H}$ NMR $\delta 11.46$ (br s, 1H), 7.43-7.41 (m, 3H), 7.36-7.32 (m, 2H), $5.49(\mathrm{~s}, 1 \mathrm{H}), 4.35$ (t, J=6.7 Hz, 2H), 3.23-3.16 (m, 2H), 1.78-1.71 (m, 2H), 1.60-1.50 (m, 2H), 1.44-1.32 (m, 2H), $0.98(\mathrm{t}, J=$ $7.4 \mathrm{~Hz}, 3 \mathrm{H}), 0.87(\mathrm{t}, J=7.4 \mathrm{~Hz}, 3 \mathrm{H}) ;{ }^{13} \mathrm{C} \mathrm{NMR} \delta 201.9,136.5,129.3,128.6,128.4,127.4,100.3$, 69.6, 44.8, 32.5, 22.0, 19.9, 13.6, 10.5. Anal. Calcd for $\mathrm{C}_{16} \mathrm{H}_{23} \mathrm{NOS}$ : C, 69.27; H, 8.36; N, 5.05. Found: C, 69.48; H, 8.51; N, 4.89 .

O-Methyl (Z)-3-(butylamino)-3-(4-pyridinyl)-2-propenethioate (5c). Yellow oil (74\%); ${ }^{1} \mathrm{H}$ NMR $\delta 11.29$ (br s, 1H), 8.73-8.70 (m, 2H), 7.27-7.26 (m, 2H), $5.40(\mathrm{~s}, 1 \mathrm{H}), 3.98(\mathrm{~s}, 3 \mathrm{H})$, $3.18-3.12(\mathrm{~m}, 2 \mathrm{H}), 1.60-1.50(\mathrm{~m}, 2 \mathrm{H}), 1.42-1.32(\mathrm{~m}, 2 \mathrm{H}), 0.89(\mathrm{t},=7.3 \mathrm{~Hz}, 3 \mathrm{H}) ;{ }^{13} \mathrm{C} \mathrm{NMR} \delta$ 162.4, 150.5, 150.2, 144.2, 122.1, 99.7, 55.4, 44.9, 32.5, 19.9, 13.6. Anal. Calcd for $\mathrm{C}_{13} \mathrm{H}_{18} \mathrm{~N}_{2} \mathrm{OS}$ : C, 62.36; H, 7.25; N, 11.19. Found: C, 62.07; H, 7.70; N, 11.50.

\section{General procedure for the preparation of $\boldsymbol{\beta}$-enamino dithioesters $6 \mathrm{a}-\mathrm{c}$}

Benzotriazolyl $\beta$-enaminothione $3(0.3 \mathrm{mmol})$ and potassium hydroxide $(0.2 \mathrm{~g})$ were dissolved in appropriate thiol $(2 \mathrm{~mL})$. The reaction mixture was exposed to microwave irradiation ( 80 Watts, $80^{\circ} \mathrm{C}$ ) for $0.5 \mathrm{~h}$. The solvent was removed under vacuum and the residue was dissolved in dichloromethane $(10 \mathrm{~mL})$, and the solution was washed with $5 \%$ aqueous $\mathrm{Na}_{2} \mathrm{CO}_{3}(3 \times 10 \mathrm{~mL})$, followed by brine $(10 \mathrm{~mL})$. The organic layer was dried over anhydrous $\mathrm{Na}_{2} \mathrm{SO}_{4}$. The solvent was removed under vacuum, and the residue purified by flash chromatography (hexanes/ethyl acetate, $5: 1)$ on silica gel to give $\mathbf{6 a}-\mathbf{c}$.

Hexyl (Z)-3-(butylamino)-3-phenyl-2-propenedithioate (6a). Light yellow oil (85\%); ${ }^{1} \mathrm{H}$ NMR $\delta 12.85$ (br s, 1H), 7.45-7.43 (m, 3H), 7.36-7.33 (m, 2H), $6.11(\mathrm{~s}, 1 \mathrm{H}), 3.26-3.16(\mathrm{~m}, 4 \mathrm{H})$, $1.72-1.51(\mathrm{~m}, 4 \mathrm{H}), 1.44-1.26(\mathrm{~m}, 8 \mathrm{H}), 0.91-0.84(\mathrm{~m}, 6 \mathrm{H}) ;{ }^{13} \mathrm{C}$ NMR $\delta 203.4,163.6,135.6$, 129.6, 128.6, 127.4, 109.6, 44.9, 33.0, 32.3, 31.4, 28.8, 28.7, 22.5, 19.9, 14.0, 13.5. Anal. Calcd for $\mathrm{C}_{19} \mathrm{H}_{29} \mathrm{NS}_{2}: \mathrm{C}, 68.00 ; \mathrm{H}, 8.71 ; \mathrm{N}, 4.17$. Found: C, 67.71; H, 9.03; N, 4.14.

Phenyl (Z)-3-(butylamino)-3-phenyl-2-propenedithioate (6b). Recrystallized from ethyl acetate/hexanes to give yellow microcrystals $(87 \%)$, mp $76-78{ }^{\circ} \mathrm{C} ;{ }^{1} \mathrm{H}$ NMR $\delta 13.16$ (br s, $\left.1 \mathrm{H}\right)$, 7.54-7.50 (m, 2H), 7.41-7.38 (m, 6H), 7.26-7.22 (m, 2H), $5.88(\mathrm{~s}, 1 \mathrm{H}), 3.27-3.21(\mathrm{~m}, 2 \mathrm{H})$, 1.60-1.50 (m, 2H), 1.43-1.32 (m, 2H), $0.85(\mathrm{t}, J=7.3 \mathrm{~Hz}, 3 \mathrm{H}) ;{ }^{13} \mathrm{C}$ NMR $\delta 201.7,165.0,135.6$, $135.2,132.2$, 129.9, 129.5, 129.2, 128.6, 127.3, 108.6, 45.2, 32.1, 19.9, 13.5. Anal. Calcd for $\mathrm{C}_{19} \mathrm{H}_{21} \mathrm{NS}_{2}$ : C, 69.68; H, 6.46; N, 4.28. Found: C, 69.78; H, 6.54; N, 4.04.

Phenyl (Z)-3-(butylamino)-3-(4-pyridinyl)-2-propenedithioate (6c). Orange oil (92\%); ${ }^{1} \mathrm{H}$ NMR $\delta 13.04$ (br s, 1H), 8.70-8.68 (m, 2H), 7.52-7.47 (m, 2H), 7.42-7.40 (m, 3H), 7.19-7.17 $(\mathrm{m}, 2 \mathrm{H}), 5.79(\mathrm{~s}, 1 \mathrm{H}), 3.21-3.14(\mathrm{~m}, 2 \mathrm{H}), 1.59-1.49(\mathrm{~m}, 2 \mathrm{H}), 1.43-1.32(\mathrm{~m}, 2 \mathrm{H}), 0.86(\mathrm{t}, J=7.3$ $\mathrm{Hz}, 3 \mathrm{H}) ;{ }^{13} \mathrm{C}$ NMR $\delta$ 204.8, 161.3, 150.3, 142.8, 135.6, 131.7, 129.8, 129.2, 121.6, 107.3, 45.1, $32.1,19.8,13.4$. 


\section{General procedure for the preparation of $\beta$-enaminothiones 8a-c}

The appropriate (6-nitrobenzotriazol-1-yl)methanethione $7 \mathbf{a}-\mathbf{c}(1.0 \mathrm{mmol})$ and $\mathrm{ZnBr}_{2}(2.0 \mathrm{mmol})$ was dissolved in THF $(20 \mathrm{~mL})$ and stirred at room temperature for $1 \mathrm{~h}$. A solution of ketimine $\mathbf{2 a}$ $(1.0 \mathrm{mmol})$ in THF $(10 \mathrm{~mL})$ was added dropwise during $5 \mathrm{~min}$, and the mixture was allowed to stir at room temperature for $\mathbf{3 d}$. The completion of reaction was monitored by TLC. The reaction was quenched with $5 \%$ aqueous $\mathrm{KOH}(20 \mathrm{~mL})$ and the product was extracted with dichloromethane $(3 \times 15 \mathrm{~mL})$. The extract was washed with brine $(2 \times 15 \mathrm{~mL})$, dried over anhydrous $\mathrm{MgSO}_{4}$ and concentrated under vacuum to give the crude product, which was purified by flash chromatography on silica gel using chloroform to give $\mathbf{8 a}-\mathbf{c}$.

(Z)-3-(Butylamino)-1-(4-chlorophenyl)-3-phenyl-2-propene-1-thione (8a). Red oil (91\%); ${ }^{1} \mathrm{H}$ NMR $\delta 14.49$ (br s, 1H), 7.68 (d, $J=8.3 \mathrm{~Hz}, 2 \mathrm{H}), 7.51-7.46(\mathrm{~m}, 3 \mathrm{H}), 7.43-7.38$ (m, 2H), 7.28 $(\mathrm{d}, J=8.3 \mathrm{~Hz}, 2 \mathrm{H}), 6.54(\mathrm{~s}, 1 \mathrm{H}), 3.37$ (q, $J=6.8 \mathrm{~Hz}, 2 \mathrm{H}), 1.70-1.61(\mathrm{~m}, 2 \mathrm{H}), 1.48-1.39(\mathrm{~m}$, 2H), $0.91(\mathrm{t}, J=7.3 \mathrm{~Hz}, 3 \mathrm{H}) ;{ }^{13} \mathrm{C}$ NMR $\delta 199.7,167.8,146.9,135.4,135.2,130.1,128.8,128.2$, 128.0, 127.3, 113.0, 45.3, 32.1, 20.1, 13.6. Anal. Calcd for $\mathrm{C}_{19} \mathrm{H}_{20} \mathrm{ClNS}$ : C, 69.18; H, 6.11; N, 4.25. Found: C, 68.92; H, 6.41; N, 3.89.

(Z)-3-(Butylamino)-1-(2-thienyl)-3-phenyl-2-propene-1-thione (8b). Red oil (76\%); ${ }^{1} \mathrm{H}$ NMR $\delta 14.04$ (br s, 1H), 7.50-7.40 (m, 7H), 7.02-6.99 (m, 1H), $6.65(\mathrm{~s}, 1 \mathrm{H}), 3.33(\mathrm{q}, J=6.4 \mathrm{~Hz}, 2 \mathrm{H})$, 1.67-1.47 (m, 2H), 1.46-1.39 (m, 2H), 0.89 (t, $J=7.3 \mathrm{~Hz}, 3 \mathrm{H}) ;{ }^{13} \mathrm{C}$ NMR $\delta 189.6,167.3,154.4$, 135.6, 130.9, 129.9, 128.8, 127.8, 127.3, 124.6, 109.9, 45.3, 32.1, 20.0, 13.5. Anal. Calcd for $\mathrm{C}_{17} \mathrm{H}_{19} \mathrm{NS}_{2}$ : C, 67.73; H, 6.35; N, 4.65. Found: C, 68.09; H, 6.48; N, 4.25.

(Z)-3-(Butylamino)-1-(2-furyl)-3-phenyl-2-propene-1-thione (8c). Red oil (45\%); ${ }^{1} \mathrm{H}$ NMR $\delta$ 14.16 (br s, 1H), 7.42-7.40 (m, 3H), 7.34-7.31 (m, 3H), $7.12(\mathrm{~d}, J=3.7 \mathrm{~Hz}, 1 \mathrm{H}), 6.72(\mathrm{~s}, 1 \mathrm{H})$, $6.36(\mathrm{dd}, J=3.4,1.5 \mathrm{~Hz}, 1 \mathrm{H}), 3.27(\mathrm{q}, J=6.4 \mathrm{~Hz}, 2 \mathrm{H}), 1.58-1.51(\mathrm{~m}, 2 \mathrm{H}), 1.39-1.31(\mathrm{~m}, 2 \mathrm{H})$, $0.82(\mathrm{t}, J=7.3 \mathrm{~Hz}, 3 \mathrm{H}) ;{ }^{13} \mathrm{C}$ NMR $\delta 183.0,167.8,158.9,143.6,135.6,129.9,128.7,127.3$, 113.9, 112.7, 109.0, 45.3, 32.1, 20.0, 13.5. Anal. Calcd for $\mathrm{C}_{17} \mathrm{H}_{19} \mathrm{NOS}: \mathrm{C}, 71.54 ; \mathrm{H}, 6.71 ; \mathrm{N}$, 4.91. Found: C, 71.28; H, 7.02; N, 4.53.

4-Chloro- $N$-phenyl-thiobenzamide (9). Was obtained from the reaction of $\mathbf{7 a}$ with imine $\mathbf{2 i}$, following the procedure for $\mathbf{8 a}-\mathbf{c}$. Recrystallization from dichloromethane/hexanes gave light yellow microcrystals $(35 \%)$, mp $153-155{ }^{\circ} \mathrm{C}$ (lit. ${ }^{30} \mathrm{mp} 157-158{ }^{\circ} \mathrm{C}$ ); ${ }^{1} \mathrm{H}$ NMR $\left(\mathrm{DMSO}-d_{6}\right) \delta$ 11.83 (br s, 1H), 7.87-7.80 (m, 4H), 7.54 (d, $J=8.1 \mathrm{~Hz}, 2 \mathrm{H}), 7.45$ (t, $J=7.7 \mathrm{~Hz}, 2 \mathrm{H}), 7.28$ (t, $J$ $=7.3 \mathrm{~Hz}, 1 \mathrm{H}) ;{ }^{13} \mathrm{C} \mathrm{NMR}\left(\mathrm{DMSO}-d_{6}\right) \delta 195.9,141.2,139.9,135.5,129.3,128.5,128.0,126.4$, 124.2 .

\section{References}

1. Cremlyn, R. J. An Introduction to Organosulfur Chemistry; John Wiley \& Sons: New York, 1996.

2. Vedejs, E.; Krafft, G. A. Tetrahedron 1982, 38, 2857. 
3. Wilde, T. G.; Billheimer, J. T.; Germain, S. J.; Hausner, E. A.; Meunier, P. C.; Munzer, D. A.; Stoltenborg, J. K.; Gillies, P. J.; Burcham, D. L.; Huang, S. M.; Klackiewick, J. D.; Ko, S. S.; Wexler, R.R. Bioorg. Med. Chem. 1996, 4, 1493.

4. Missio, L. J.; Braibante, H. S.; Braibante, M. E. F. J. Heterocycl. Chem. 1996, 33, 1243.

5. Palacios, F.; Aparicio, D.; Garcia, J. Tetrahedron 1997, 53, 2931.

6. Al-Badri, H.; Collignon, N.; Maddalino, J.; Masson, S. Tetrahedron 2000, 56, 3909.

7. Bogdanowicz-Szwed, K.; Budzowski, A. Monatsh. Chem. 2001, 132, 947.

8. Coen, S.; Ragonnet, B.; Vieillescazes, C.; Roggero, J. Heterocycles 1985, 23, 1225.

9. Meslin, J. C.; Reliquet, A.; Reliquet, F.; Quiniou, H. Synthesis 1980, 453.

10. Cativiela, C.; Serrano, J. L.; Zurbano, M. M. J. Org. Chem. 1995, 60, 3074.

11. Barluenga, J.; Gonzalez, F. J.; Gotor, V.; Fustero, S. J. Chem. Soc., Perkin. Trans. 1 1988, 1739.

12. Adiwidjaja, G.; Proll, T.; Walter, W. Tetrahedron Lett. 1981, 22, 3175.

13. Kascheres, A.; Kascheres, C.; Braga, A.C.H. J. Org. Chem. 1993, 58, 1702.

14. Saito, T.; Takekawa, K.; Takahashi, T. Chem. Commun. 1999, 1001.

15. Sosnicki, J. G.; Jagodzinski, T. S.; Hansen, P. E. Tetrahedron 2001, 8705.

16. Cava, M. P.; Levinson, M. I. Tetrahedron 1985, 41, 5061.

17. Albert, R.; Knecht, H.; Andersen, E.; Hungerford, V.; Schreier, M. H.; Papagerogiou, C. Bioorg. Med. Chem. Lett. 1998, 8, 2203.

18. Braibante, M. E. F.; Braibante, H. S.; Missio, L.; Andricopulo, A. Synthesis 1994, 898.

19. Ramadas, S. R.; Srinivasan, P. S.; Ramachandran, J.; Sastry, V. V. S. K. Synthesis 1983, 605.

20. Orth, R. E.; Soedigdo, S. J. Heterocycl. Chem. 1965, 2, 486.

21. Larsen, C.; Steliou, K.; Harpp, D. N. J. Org. Chem. 1978, 43, 337.

22. Smith, J. K.; Bergbreiter, D. E.; Newcomb, M. J. Am. Chem. Soc. 1983, 105, 4396.

23. Boduszek, B. Tetrahedron 1996, 52, 12483.

24. Asinger, F.; Offermanns, H.; Köhler, H.-D. Tetrahedron Lett. 1967, 8, 631.

25. Liao, S.; Collum, D. B. J. Am. Chem. Soc. 2003, 125, 15114.

26. Paulvannan, K.; Stille, J. R. J. Org. Chem. 1992, 57, 5319.

27. Baricordi, N.; Benetti, S.; Biondini, G.; De Risi C.; Pollini, G. P. Tetrahedron Lett. 2004, 45, 1373.

28. Varma, R. S.; Dahiya, R. Tetrahedron 1998, 54, 6293.

29. Katritzky, A. R.; Witek, R. M.; Rodriguez-Garcia, V.; Mohapatra, P. P.; Rogers, J. W.; Cusido, J.; Abdel-Fattah, A. A. A.; Steel, P. J. J. Org. Chem. 2005, 70, 7866.

30. Waisser, K.; Houngbedji, N.; Machacek, M.; Sekera, M.; Urban, J.; Odlerova, Z. Collect. Czech. Chem. Commun. 1990, 55, 307. 